\title{
Asymmetric Synthesis of (-)-Indolizidine 209D via B-Alkyl Suzuki Coupling and Amination Reactions
}

\author{
Guncheol Kim," Jae Hak Shim, and Jin Hee Kim \\ Department of Chemistr?. College of Natural Science, Chungham National liniversity. Daejeon 305-76-. Korea \\ Received September 16, 2003
}

Key Words : Indolizidine 209D, B-Alkyl Suzuki coupling, Furan, Reductive amination

The indolizidine alkaloids have been isolated from the skin secretions of neotropical frogs, ${ }^{1}$ and some have been shown to function as non-competitive blockers for inuscletype and ganglionic nicotinic receptor channels. The simplest bicyclic gephyrotoxin alkaloid Indolizidine 2090 possessing a single substituent at $\mathrm{C} 5$ of the indolizidine skeleton." has been isolated in the limited amount of these compounds available from natural sources, and the pharmacological interest has made it an attractive synthetic subject. $^{4}$

Recently, we reported the synthesis of (-)-indolizidine $209 \mathrm{D}$ via B-alkyl Suzuki coupling and amination reaction. and the utilization of furan for 1,3-dicarbonyl moiety for $(+)$ monomorine synthesis. As an extension, we wanted to suggest a new way for the concise synthesis of (-)indolizidine $209 \mathrm{D}$. For the purpose, we considered that intermediate 1 would be the proper precursor, as the mild hydrogenation condition was expected to induce the consecutive reductive amination reaction as well as hydro- genation of double bond to afford the final product. In this course, stereochemistry of the chiral center in $\mathbf{3}$ would determine the delivery of hydrogen atom at the developing tetrahedral center at $\mathrm{C} 9$ from the least hindered site with respect to hexyl group. 5

The intermediate $\mathbf{3}^{6}$ was readily prepared by the reported route and the 2-iodofuran 4 was obtained by slight modification of the known procedure. ${ }^{4 i, 7}$ The Suzuki coupling ${ }^{8}$ of 3 and 4 provided the required intermediate 2 in $81 \%$ yield. Oxidative opening of the furan ring in 2 was accomplished with NBS in $\mathrm{THF}$-acetone at $-20{ }^{\circ} \mathrm{C}$ to yield $71 \%$ of $\mathrm{I}$. Finally, the precursor I was smoothly converted to (-)indolizidine 2090 2 under 1 atm of $\mathrm{H}_{2}$ in $10 \mathrm{~h}$ at $\mathrm{rt}$ and in $63 \%$ yield. The spectral data (' $\mathrm{H}$ and MS) were identical to those reported ${ }^{\text {t: }}$.

In conclusion, we described the asymmetric synthesis of (-)-indolizidine 209D employing a new consecutive reductive amination pathway using keto-aldehyde moiety. 2lodofuran was employed for the $B$-alkyl Suzuki reaction and

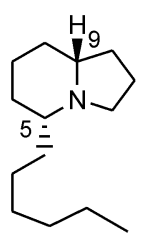

(-)-Indolizidine 209D

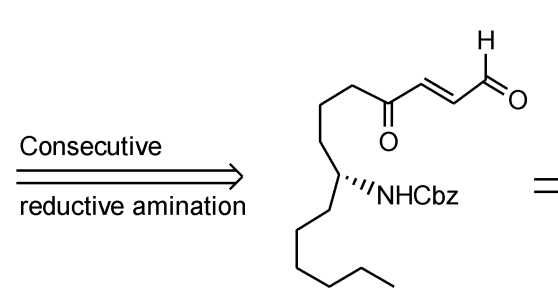

1

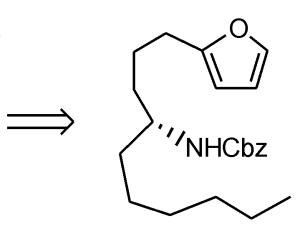

2

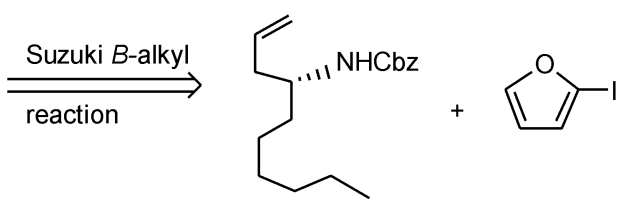

3

4

Scheme 1

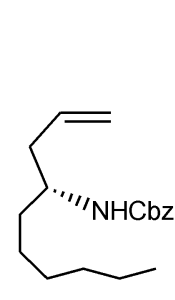

3

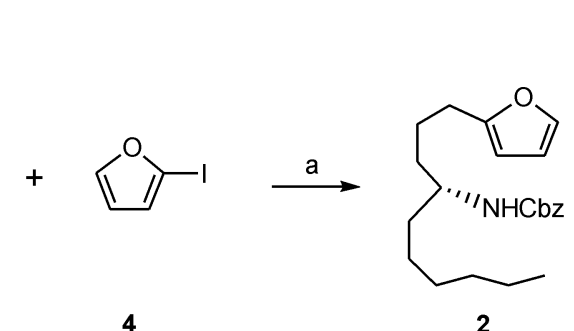

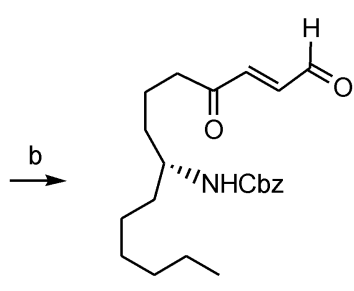

1

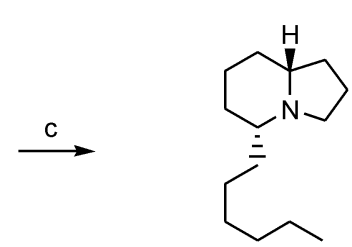

(-)-Indolizidine 209D

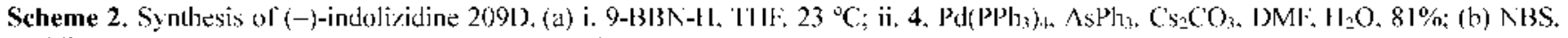
Pyridine. TIIF-Acetone. -20 'C. $71 \% ;$ (c) 11 . $10 \%$ Pd-C. MeOII. $63 \%$. 
afterward transformed to the proper precursors.

\section{Experimental Section}

General for the selected experiments. ${ }^{1} \mathrm{H}$ NMR spectra were recorded on a Bruker AC 200 spectrometer using tetrantethylsilane as an internal standard. Chemical shifts are measured in part per million(d) and coupling constants, $J$. are reported in $\mathrm{Hz}$. All reactions were carried out under nitrogen atmosphere and anlydrous solvents were used.

2-Iodofuran 4. $n$-BuLi $(5.8 \mathrm{~mL}$. $14.6 \mathrm{mmol}$ of $2.5 \mathrm{M}$ in hexane) was added slowly to a solution of furan $(1.1 \mathrm{~mL}$. $14.6 \mathrm{~mm}$ ol $)$ in dry ether $(10 \mathrm{~mL})$ at $-78^{\circ} \mathrm{C}$. The solution was warmed to $0^{\circ} \mathrm{C}$. To this solution was added slowly iodine (3.7 g. $14.6 \mathrm{mmol}$ ) in $10 \mathrm{~mL}$ of dry ether via syringe. The resulting solution was warmed to $0{ }^{\circ} \mathrm{C}$ using ice bath and stirred for $2.5 \mathrm{hr}$. After quenching the reaction with $30 \mathrm{~mL}$ of water. the reaction mixture was extracted diethyl ether (70 $\mathrm{mL} \times 2$ ). The organic layer was washed with $30 \mathrm{~mL}$ of aqueous $\mathrm{Na}_{3} \mathrm{~S}_{2} \mathrm{O}_{3}$ solution, water, brine, and dried over $\mathrm{MgSO}_{4}$. After filtration, the organic layer was concentrated to yield $2.27 \mathrm{~g}$ of relatively pure 2 -iodofuran 4 ( $80 \%$ yield): ${ }^{1} \mathrm{H}$ NMR $\left(80 \mathrm{MHz} . \mathrm{CDCl}_{3}\right) \delta 6.29(\mathrm{~m}, \mathrm{lH}), 6.50(\mathrm{~m}, \mathrm{lH})$. $7.50(\mathrm{nt}, \mathrm{lH})$

4-Benzyloxycarbonylamino-1-(2-furyl)-decane 2. To a solution of $N$-Cbz-homoallylic anine $(0.50 \mathrm{~g} .1 .735 \mathrm{mumol})$ in THF (15 mL) was added 9-BBN (637 $\mathrm{mg} .2 .606 \mathrm{mmol})$ in $1 \mathrm{~mL}$ of THF under Ar. The solution was stirred at it for $2 \mathrm{hr}$. and to this solution was added water $(310 \mathrm{mg} .17 .35 \mathrm{~nm}$ ol). The resulting solution was transferred by syringe to a solution of 2-iodoform (670 $\mathrm{mg}, 3.47 \mathrm{mmol}$ ) in DMF (25 $\mathrm{mL})$ containing $\mathrm{Pd}\left(\mathrm{PPh}_{\hat{j}}\right)_{4}(200 \mathrm{mg}, 0.173 \mathrm{mmol}), \mathrm{Ph}_{j} \mathrm{As}(53$ mg, $0.173 \mathrm{mmol}$ ) and water $(310 \mathrm{mg} .17 .35 \mathrm{mmol}$ ) which had been stirred for 1 hr. The combined mixture was stirred at It overnight. After dilution of the solution with $20 \mathrm{~mL}$ of EtOAc. the organic layer was washed with saturated $\mathrm{NH}_{4} \mathrm{Cl}$ solution $(30 \mathrm{mLL})$, saturated $\mathrm{NaHCO}_{3}$ solution, water (20 $\mathrm{mL}$ ). and brine. The organic layer was dried over $\mathrm{MgSO}_{4}$. filtered through celite and concentrated. The crude product was separated by columun chromatography (hex : EtOAc = $10: 1$ ) to yield $507 \mathrm{mg}$ of compound $2(81 \%)$ : IR (thin film) $3324,2934,2853,1685,1541 \mathrm{~cm}^{-1}$ : ${ }^{1} \mathrm{H}$ NMR $(300 \mathrm{MHz}$. $\left.\mathrm{CDCl}_{3}\right) \delta 0.85(\mathrm{t} . J=6.2 \mathrm{~Hz}, 3 \mathrm{H}), 1.23-1.69(\mathrm{~m} .16 \mathrm{H}), 2.58-$ $2.62(\mathrm{~m} .2 \mathrm{H}) .3 .61(\mathrm{~m} .1 \mathrm{H}) .4 .44$ (d. $J=8.90 \mathrm{~Hz}, 1 \mathrm{H}) .5 .07$ (s. 2H) $5.94(\mathrm{~m}, 1 \mathrm{H}), 6.24(\mathrm{~m}, \mathrm{lH}) .7 .26(\mathrm{~m}, 1 \mathrm{H}) .7 .33(\mathrm{~m}$. $5 \mathrm{H}):{ }^{13} \mathrm{C} \mathrm{NMR}\left(100 \mathrm{MHz}, \mathrm{CDCl}_{3}\right) \delta 14.1,22.6,24.3,25.7$. 27.7. 29.2. 31.7. 34.8, 35.4. 51.1.66.5, 104.9. 110.2. 128.0 . $128.5,136.7,140.8,155.9,156.1$ : HRMS $\mathrm{C}_{22} \mathrm{H}_{31} \mathrm{NO}_{3}$. 357.2304 (cald. 357.2304 )

\section{8-Benzyloxycarbonylamino-4-0x0-trans-penta-2-decenal} 1. To a solution furan compound 2 (100 mg. $0.28 \mathrm{mg}$ ) in a solution of acetone- $\mathrm{H}_{2} \mathrm{O}(10: 1,1 \mathrm{~mL})$ was added NBS (75 mg. $0.42 \mathrm{mmol}$ ) at $-15^{\circ} \mathrm{C}$. After the mixture was stirred at $-15^{\circ} \mathrm{C}$ for 40 mins pyridine $(0.10 \mathrm{~mL})$ was added and the resulting solution was stirred at $\mathrm{rt}$ for $2 \mathrm{hr}$. After dilution of the mixture with $10 \mathrm{~mL}$ of EtOAc the organic layer was washed with $1 \mathrm{~N} \mathrm{HCl}$ solution and dried over $\mathrm{MgSO}_{4}$.
Filtration was followed by concentration and silica-gel columu chromatography (hex: EtOAc $=1: 1$ ) to afford a yellowish product ( $75 \mathrm{mg} .71 \%$ ): IR (thin film) 3328.2932 , 2853. $1687,1538 \mathrm{~cm}^{-1}$. ${ }^{1} \mathrm{H}$ NMR $\left(300 \mathrm{MHz}, \mathrm{CDCl}_{3}\right) \delta 0.86$ (t. $J=6.3 \mathrm{~Hz} .3 \mathrm{H}$ ), 1.23-1.71 (m. 16H). 2.61-2.75 (m, 2H), $3.62(\mathrm{~m}, \mathrm{HH}) .4 .50(\mathrm{~d}, J=8.90 \mathrm{~Hz} . \mathrm{HH}) .5 .08(\mathrm{~s}, 2 \mathrm{H}), 6.83(\mathrm{~d}$, $J=16.2 \mathrm{~Hz}, 1 \mathrm{H}), 6.74$ (dd. $J=16.2 \mathrm{~Hz}, J=6.7 \mathrm{~Hz} .1 \mathrm{H})$, 7.29 (m. 5H), 9.73 (d. $J=6.7 \mathrm{~Hz}, 1 \mathrm{H}) ;{ }^{13} \mathrm{C}$ NMR $(100 \mathrm{MHz}$, $\left.\mathrm{CDCl}_{3}\right) \delta$ 14.6. 19.6. 22.5. 25.7. 29.1. 31.7. 34.6, 35.4, 40.9. 50.7. 66.5. 127.9. 128.1. 128.5, 136.6, 137.4, 144.7, 156.2, 193.4. 199.7: HRMS C ${ }_{23} \mathrm{H}_{31} \mathrm{NO}_{4} .373 .2247$ (cald. 373.2253).

(-)-Indolizidine 209D. A solution of 1 in $\mathrm{MeOH}(2 \mathrm{~mL})$ was stirred with $10 \%$ palladium on activated carbon under latm of $\mathrm{H}_{2}$ at rt for $10 \mathrm{hr}$. After dilution with $\mathrm{Et}_{2} \mathrm{O}(5 \mathrm{~mL})$, the mixture was filtered through celite and concentrated to afford $10 \mathrm{mg}$ of $(-)-209 \mathrm{D}(65 \%):[\alpha]_{\mathrm{D}}^{2+}-77.0(c 0.80$. $\left.\left.\mathrm{CH}_{2} \mathrm{Cl}_{2}\right)\left[\mathrm{lit}^{+{ }^{+c}}[\alpha]_{\mathrm{D}}^{20}-89.64(c) 1.880 . \mathrm{CH}_{2} \mathrm{Cl}_{2}\right)\right]$.

Acknowledgements. This work was supported by grant (R01-2000-000-00048-0) from the Basic Research Program of Korea Science and Engineering Foundation and we appreciate Center for Research Facilities. CNU for the pernnission to NMR.

\section{References}

1. Tokuryama, T; Nishimori. N.: Karle, I. L.; Edwards. M. W.; Daly: J. W. Tetrahedron 1986. +2. 3453 .

2. Aronstam. R. S.: Daly. T. W.: Spande. T. F.: Narayanan. T. K.: Albuquerque. E. X. Newrochen. Res. 1986. HI. 1227.

3. Their structures have been tentatively assigned on the basis of the mass spectrum [Daley, J. W. Fortsch: Chem. Org. Naturst. 1982. 11. 205].

4. For asymmetric syntheses of indolizidine 209D. see the following references. (a) Ånmant. T.: Somfai. P. Tetrohedon Lett. 1995. 36. 303. (b) A himan1. T.: Somfai. P. Tetrohedron 1995. 51. 9747. (c) Nukui. S.: Sodeoka. M.: Sasai. H.; Shibasaki. M. J. Org. Chent. 1995. 60,398. (d) Jefford. C. W. Sienkiewicz, K.: Thornton, S. R. Helv Chim Acta 1995. 78. 1511. (e) Takahata, H.; Kubota, M: Ihara. K.: Okamoto. N.: Momose. I.: Azer. N.: Eldefrawi. A. T.: Eldefrawi. M. E. Tetrahedon: Astnmetry 1998. 9. 3289. (f) Chệnevert. R.: Ziaranii. G. M.: Morin. M. P.: Dasser. M. Tetrahedon: Asymmetry 1999. 10,3117. (g) Yamazaki, N.; Ito. T.; Kibayashi, C. Org. Lett 2000, 2. 465. (h) Back. T. G.; Nakajima. K. J. Org. Chem 2000. 65,4543. (i) Kim, G.: Jung. S.-d.: Kim. W.j. Org. Lett. 2001.3 .2985$.

5. (a) Jefford. C. W. Tang. Q.: Zaslona. A. J. Am. Chent. Soc. 1991. 113. 3513. (b) Robins. D. J.: Sakdaret. S. J. Chent. Soc. Perkins Trans. $] 1981,909$

6. Yu. C.-M.: Choi. H.-S.; Yoon. S.-K.: Jung. W.-H. Symlett 1997. 889. The enantiomeric purity was determined to be $292^{\circ}$ o via ${ }^{1} \mathrm{H}$ NMR using Eu(hfe $)_{3}$ in $\mathrm{CDCl}_{3}$.

7. Carmen1. C. S.: Koser. G. F. J. Org Chent 1983. 48. 2534

8. (a) Miyamura. N.: Suzuki. A. Chem. Rev 1995. 95. 2457. (b) Suzuki. A. J. Organomet. Chent 1999. 576. 147. For recent application to natural product syntheses, see the following references. (c) Onba, M.: Kawase. N.: Fujii. T. J. Am Chem Soc. 1996. 118. 8250 (d) Su. D.-S.: Sorensen. E. T.: Danishefsky. S. J. Angew: Chem. In. Ed. Engl. 1996. 35. 2801. (e) Takemoto. T: Sodeoka. M.: Sasai. H.: Shibasaki. M. J. Org Chem 1996. 61. 4876. (f) Meng, D.; Bertinato, P.: Balog. A.; Su. D.-S: Kamenecka. T.: Sorensen. E.; Danishefsky. S. J. J. Am. Chem. Soc. 1997. 119, 10073. (g) Su, D.-S.; Balog, A.: Meng, D.: 
Bertinato. P.: Danishefsky, S. J.: Zheng. Y.-H.: Chou. T.-C.: He, L.: Horwitz. S. B. Angew. (Chem. Iht Ed. Engl. 1997. 36. 2093. (h) Fürstner. A.: Seidel. G. J. Org Chent 1997. 62.2332. (i) Trost. B. M.: Lee. C. B. J. Am. Chem. Soc. 1998. 120.6818. (j) Balog. A.: Harris. C.: Savin, K.: Zhang. G. Chou, T.-C.: Danishefsky. S. I. Angew: Chem. Int. Ed. Engl. 1998, 37, 2675. (k) Fürstner A.: Konetzki. I. J. Org. (Them. 1998. 63. 3072. (1) Sasaki. M. Fuwa. H.: Inoue. M.: Tachibana. K. Tetrahedron lett. 1998. 39.9027. (m) Meng. D.: Danishefsky. S. J. Angew. Chem. Int. Ed. Engl. 1999. 38. 1485. (n) Meng. D.: Tan. Q: Danishetshy. S. J. Anger: Chent. Int. Ed. Engl. 1999, 38, 3197. (o) Tralner. D.: Schwartz, I. B: Danishefsky, S. I. Angew: Chem. Iht. Ed. Engl. 1999. 38. 3542. (p)
Trauner. D:- Danishefsky, S. J. Tetrahedron lett. 1999. 40,6511 (q) Harris. C. R.: Kuduk. S. D.: Balog. A.: Savin. K.: Glunz. P. W: Danishefsky. S. I. J. Am. Chent. Soc 1999. 121. 7050 (r) Zhu. B. Panek. I. S. Ong. Lett. 2000. 2. 2695. (s) Lee. C. B.: Choul. T.-C. Zhang. X.-G.; Wang, Z.-G.: Kuduk, S. K.: Chappell, M; Stachel. S. J.: Danishefskv: S. J. J. Org. Chem. 2000. 65, 6525. (t) Sasaki. M:- Noguchi, K: Fuwa, H.: Tachibana. K. Tetrahedron Lett. 2000. 11. 1425. (u) Fuwa. H.: Sasaki. M.: Tachibana. K. Tetrahedron Lett. 2000. H. 8371. (v) Nakamura. T.: Shiozaki. M. Tetrahedon Lett. 2001. t2. 2701. (w) Takakura. H.: Noguchi. K.: Sasaki. M. Tachibana. K. Angew. Chem. Int. Ed. Engl 2001. 40. 1090. 